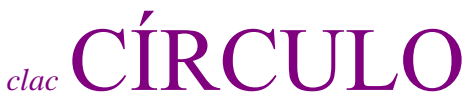 \\ de \\ lingüistica
aplicada a la \\ comunica
}

$57 / 2014$

\section{EVOLUCIÓN Y DESARROLLO DEL TEXTO DE ESPECIALIDAD}

\author{
David J. Sánchez Jiménez
}

Universidad de Washington

djsan en uw edu

\section{Resumen}

La escritura evoluciona con la sociedad y el texto lo hace dentro de cada comunidad discursiva de forma específica. Las disciplinas que se han ocupado de analizar los textos escritos se hacen eco de estos cambios y explican cuáles son las adaptaciones que siguen en esta transformación. En el presente artículo se parte de la concepción del texto desde los inicios del Análisis del Discurso para observar cómo este concepto ha evolucionado dentro de cada comunidad disciplinaria de mano de los distintos cambios que se han venido produciendo en la sociedad durante las últimas décadas.

Palabras clave: análisis textual, fines específicos

Sánchez Jiménez, David J. 2014.

Evolución y desarrollo del texto de especialidad.

Círculo de Lingüística Aplicada a la Comunicación 57, 113-136.

http://www.ucm.es/info/circulo/no57/sanchez.pdf

http://revistas.ucm.es/index.php/CLAC

DOI: http://dx.doi.org/10.5209/rev_CLAC.2014.v57.44517

(C)2014 David J. Sánchez Jiménez

Círculo de Lingüística Aplicada a la Comunicación (clac)

Universidad Complutense de Madrid. ISSN 1576-4737. http://www.ucm.es/info/circulo 


\begin{abstract}
Evolution and development of language for specific purposes texts.

Writing volves in society and so do kinds of text in a discourse community in specific ways. The disciplines involved in the analysis of these written texts follow these changes and explain these adjustments. This article reviews the idea of texts when discourse analysis began. Its objective is to look at how this concept has evolved within each discourse community through the several changes that have occurred in the society during the last few decades.
\end{abstract}

Key words: textual analysis, specific purposes

Índice

Resumen 113

Abstract 114

1. Punto de partida 114

2. Diversificación de las disciplinas y géneros 118

3. Ampliación conceptual del texto a partir del análisis de género 121

4. La especialización de las lenguas con propósitos específicos 125

5. Conclusiones 132

Bibliografía 132

1. Punto de partida

El término Análisis del Discurso (AD) es empleado por primera vez por Zellig Harris (cit. Alcaraz y Martínez, 2004: 55) en 1952 para "aludir al análisis de la cadena hablada 
desde un punto de vista comunicativo y también para relacionar los términos cultura y lengua" (Alcaraz y Martínez, 2004: 55). Como disciplina, el AD se desarrolla en la década de los setenta y desde entonces, como señala Hyland (Matsuda y otros, 2003: 165), ha sido una herramienta esencial para conocer el uso del lenguaje en situación a partir de la exploración de los textos y de la información que aporta sobre el propósito, la función del lenguaje en uso y de las restricciones que operan en los emisores en cada contexto. El AD supera en su evolución la simple descripción microestructural del texto desde un punto de vista lingüístico para referirse a aspectos más amplios y complejos, al considerar los contextos social y cultural en el uso de la lengua. Se trata de una disciplina multidisciplinar que se ha ido desarrollando dentro de una variedad extensa de enfoques, hecho motivado por un amplio grado de intereses y orientaciones, siendo las tres disciplinas que más influyen en su desarrollo en distintos momentos de su evolución la Sociología, la Psicología Cognitiva y la Lingüística (Bhatia, 1993: 3).

Esta perspectiva poliédrica es la que permitirá estudiar el texto en su totalidad desde sus valores macrotextuales, su función comunicativa y los patrones típicos del discurso, pues el $\mathrm{AD}$ trabaja con la conexión que tiene lugar entre el lenguaje que empleamos en un contexto concreto, la sociedad en la que vivimos y los procesos mentales que intervienen cuando nos comunicamos con otros hablantes. Ya desde los lingüistas funcionalistas, como Halliday (1973, 1985), Hasan (1989) y Martin (1992), se considera la lengua como un recurso cuya finalidad básica es la de construir significado, en lugar de un conjunto de normas. Estos autores introducen la idea de que el individuo hace una selección precisa del uso de la lengua según el género y la estructura del texto con el que trabaja, motivo por el cual se puede hablar de géneros específicos ligados a la cultura a la que este pertenece y con unas características propias y concretas que están fuertemente vinculadas con la cultura y el contexto social en los que se desarrollan (Oliver del Olmo, 2004: 25-26).

El desarrollo histórico del campo de la Lingüística y del AD indica que el análisis textual ha progresado en los últimos cincuenta años desde la pura descripción lingüística a una descripción profunda de varios aspectos del texto o del género estudiado. Ligado a esta evolución, se observa también un proceso de desarrollo paralelo por el cual ha pasado de fijarse la atención en los rasgos léxico-gramaticales para centrarse en los 
aspectos retóricos que se relacionan con la organización del discurso, la cual variará en función del propósito comunicativo que quiera transmitir el emisor del mensaje en cada momento (Bhatia, 1993: 11-12). Bhatia (2008: 161) señala cómo estas descripciones se aplicaban al estudio de las lenguas con propósitos específicos en los años setenta y ochenta, cuando aún apenas se tenía en cuenta el análisis del contexto. Este estaba básicamente restringido a la descripción de los usos de las lenguas relacionados con disciplinas específicas. Tanto la enseñanza como el aprendizaje de lenguas con propósitos académicos se centraba en conceptos relacionados con la investigación lingüística a partir del análisis de texto y en los recursos lingüísticos propiamente dichos. En este sentido, comenta Swales (1990: 2) que el análisis lingüístico de las lenguas con propósitos específicos comienza con el estudio de la variedades funcionales de la lengua: "Historically, language analyses for specific purposes began in quantitative studies of the linguistic properties of functional varieties or registers of a language" (Swales: 1990: 2). De hecho, comenta este autor que el mismo concepto de género proviene de las teorías funcionalistas del lenguaje de Halliday, introducidas en su libro de 1985 An introduction to functional grammar. Esta teoría considera la relación entre el lenguaje y las funciones sociales. En base a esto, se sostiene la idea de que el ser humano en la sociedad moderna ha desarrollado formas específicas de usar el lenguaje para conseguir sus propósitos, lo que implica que los textos estén estrechamente ligados a los contextos sociales, así como a otros textos (Hyland, 2000, 2003). Desde ahí, desde lo lingüístico y lo formal, el estrecho punto de vista que partía desde una óptica textual se fue ampliando en el análisis textual en distintos niveles lingüísticos, creciendo el interés hacia los propósitos retóricos $-\mathrm{y}$ las diferencias entre las partes de un artículo-, así como hacia las diferencias retóricas que van más allá del estilo y que tienen que ver con la efectividad de la comunicación que se produce. A estas alturas se ha avanzado ya en el AD y se toman en cuenta en estas fechas otras consideraciones, al percatarse de que la observación del análisis lingüístico ya no es suficiente para conocer un texto, por estar muy limitado, como comenta Hyland (Matsuda y otros, 2003: 166), uno de los mejores conocedores de esta materia:

DA does not just look at how sentences fit together; it tries to show how they are related to their contexts. This means linking discourse features to 
issues of writer purpose, identity, audience expectations, cultural schemata, disciplinary perceptions, and so on (Matsuda y otros, 2003: 166).

Este camino se irá nutriendo paulatinamente con aportaciones de otras disciplinas emergentes como la Pragmática o la Sociolingüística, donde encuentran un lugar destacado los trabajos de Van Dijk $(1977,1981)$.

En resumen, con el establecimiento del $\mathrm{AD}$ en los años setenta como campo de estudio, las lenguas con propósitos específicos se vienen abordando desde diversos enfoques, desde los más tempranos asociados con elementos gramaticales de la comunicación científica, con los ejemplos de Barber (1962) y Swales (1986, 1990), hasta el análisis retórico y la organización discursiva en los setenta y ochenta con figuras destacadas como Lackstrom (1981), Tarone (1981), Trimble (1985) y Huckin (1986). Ya en los ochenta, en el discurso escrito de especialidad, no sólo empezaba a existir un interés por caracterizar los rasgos lingüísticos, sino también los comunicativos, incorporándose las valiosas aportaciones hechas desde los estudios nociofuncionales hasta el enfoque dominante del Análisis de Género a partir de los noventa con Swales (1990), Bhatia (1993) y Hyland (2000, 2002) como máximos representantes. El AD se diversifica entonces y pasa a estudiar también las relaciones que emparentan el discurso con la sociedad, tomando una posición política e interdisciplinar sobre aspectos como el racismo, el sexismo o la pobreza, en lo que se ha dado en llamar el análisis crítico del discurso (Van Dijk, 1993).

Todos estos hitos descritos anteriormente marcan no sólo un movimiento de lo viejo a lo moderno, sino también de la progresión cualitativa de un estudio basado en cuestiones más generales a otras más específicas, desde la superficie hasta la profundidad del lenguaje usado desde tres aspectos claramente diferenciados: los valores de los rasgos lingüísticos, la interacción entre el escritor y el lector y la atención otorgada a la estructuración del discurso (Bhatia, 1993: 17). 
2. Diversificación de las disciplinas y géneros

Se ha observado a lo largo del apartado anterior que el AD es un fenómeno dinámico y permeable al cambio, debido específicamente a su concepción multidisciplinar y por su adaptación a las evoluciones surgidas en los distintos contextos profesionales y su plasmación textual. Entre esta capacidad para evolucionar y progresar señalaba Bhatia (1993) los siguientes elementos en su avance:

In order to move towards a thicker description, discourse analysis needs a model which is rich in socio-cultural, institutional and organizational explanation, relevant and useful to language teachers and applied linguists rather than to grammatical theorists, and discriminating enough to highlight variation rather than uniformity in functional language use; a model which is not seen as an extension of grammatical formalism but is truly applied in nature, in the sense that it requires minimum support and interference from grammatical theory, and exploits maximally the conventional aspects of language use (Bhatia, 1993: 11).

Este dinamismo en dicha disciplina se extiende hasta el presente. En parte por el surgimiento de nuevo conocimiento y por la dispersión del ya existente se establecen continuamente nuevos márgenes entre las disciplinas. De este modo, los cambios que se producen en la sociedad repercuten directamente en las comunidades discursivas, y con ellas evolucionan también las diferentes características estructurales con las que se conforman los géneros (Anson, 1988: 2):

with the increasing proliferation of new knowledge, the natural separation of specialized disciplines, each with its own assumptions, methods, and linguistic characteristics, was not only inevitable but irreversible (Anson, 1988: 2)

Desde finales de los ochenta, como comenta Swales (2004: 3), vienen apareciendo composiciones interdisciplinarias, en lo que radica la dificultad para diferenciar claramente los textos académicos del discurso científico. Los lingüistas reparan entonces en que los textos producidos en distintos ambientes académicos, de negocios o 
profesionales, tienen características particulares que distinguen unos textos de otros. Swales (1990) inicia y fomenta el análisis de las secciones de los textos en varias disciplinas, e introduce el concepto de paso (move, en inglés), que define como "elements that make a paper coherent to genre-experienced readers" (Swales, 1990: 190). Para Dudley-Evans (2000) la organización de los pasos de Swales (1990) sugiere que las consideraciones retóricas gobiernan la elección de la gramática. Por lo tanto, para que el texto resulte coherente y efectivo, deberá respetar una serie de expectativas y normas convenidas por las diferentes comunidades discursivas sobre la forma de estos escritos (Dudley-Evans, 2000: 9-10).

The initial work on Move and Step analysis (Swales, 1981 and 1990; Bhatia, 1993) suggested that the models proposed were generalized models that applied to academic articles written in all academic fields. Clearly it was acknowledged that there were some differences between disciplines, but it was argued that the models proposed, such as the CARS model for the article introduction, are prototypes and actual examples will vary in the degree to which they conform with this prototype.

What is becoming clear, however, is that disciplinary variation is much more significant than allowed for in the original work on genre analysis. We need to devise a theory that goes beyond the ideas of prototypicality to acknowledge that variation in the discourse structuring of genres reflecting different epistemological and social practices in disciplines is a key factor in genre theory. The danger is that the theory will become immensely complicated with a proliferation of genres for each discipline (DudleyEvans, 2000: 9-10).

El Análisis del Género (AG) aplicado es un muy significativo desarrollo dentro del AD en su etapa más reciente, en el que un amplio número de investigadores desde una gran variedad de disciplinas han venido investigando desde el último cuarto del siglo XX. A la formalización de la teoría del AG han contribuido estudiosos de la Lingüística Teórica y Aplicada, la Sociología, incluyendo la Etnografía y la Etnometodología, la Psicología, tanto Cognitiva como los estudios aplicados y la investigación en Comunicación (Bhatia, 1993: 11). De entre todas estas disciplinas, guardan un lugar 
privilegiado los factores transculturales estudiados por la Retórica Contrastiva, que a partir de la identificación de las distintas convenciones retóricas, influencian la concreción y el entendimiento de los géneros específicos. En definitiva, esta gran variedad de disciplinas son requeridas para interpretar, describir y explicar racionalmente los distintos géneros profesionales y académicos existentes en las disciplinas.

En relación a esto, en el simposio celebrado en 2002 en la reunión del American Association en Utah, Hyland (Matsuda y otros, 2003) advierte que quedan por explorar aún muchas áreas en el discurso de L2 y que los datos con los que contamos en AD son aún limitados $\mathrm{y}$, en ningún caso concluyentes, por lo que propone tres vías de análisis para el futuro (Matsuda y otros, 2003: 169):

First, we need to consider areas of learner preference and difficulty. We know little about the lexical, syntactic, or rhetorical features of writing by particular learners in a range of different genres and professional and institutional contexts. We also require research on the extent to which these features differ from those of other learners or native speakers, and investigations into whether patterns can be explained by proficiency, by L1 conventions, or by cultural assumptions. Second, we should study areas of overlap and difference. DA research has the potential to expand our understanding of the hybridity and heterogeneity of communities and cultures, revealing the ways that communities influence writing and how these vary. Third, we can focus on areas of change and manipulation. This concerns questions of generic integrity and flexibility, the extent to which individuals can successfully challenge the conventions or ethos of theL 2 by manipulating its discourse conventions. DA can tell us more about the ways L2 writers draw on their vernacular rhetoric and what is required for these features to become recognized as new conventions (Matsuda y otros, 2003: 169).

Estas vías que señala Hyland (Matsuda y otros, 2003) sugieren nuevos caminos de investigación y muestran lo mucho que queda por hacer en este terreno. No obstante, estas líneas de investigación, como se comentará en el apartado 4 de este artículo, se 
complementan con otras áreas en un panorama cambiante. Esto sucede por la incorporación de las nuevas tecnologías y los efectos de la globalización, con intercambios de texto entre culturas que leen distintos tipos de escritos y que cuentan con convenciones lingüísticas diferentes y en diversas lenguas, factores a los que también están expuestas las lenguas con propósitos específicos a los que derivan los estudios de análisis de género.

\section{Ampliación conceptual del texto a partir del análisis de género}

Hyland (2003: 18) recuerda que el concepto de género -como pilar central de las lenguas de especialidad- es una noción que parte de las teorías funcionalistas del lenguaje descritas por Halliday en 1973 con Explorations in the functions of language y revisadas en 1985 en An introduction to functional grammar. Sin embargo, la definición de este lingüista ha sido perfilada, enriquecida y ampliada en posteriores definiciones, como la que enuncia Swales (1990) y en la que cobra especial relevancia el contexto situacional y cultural de uso del lenguaje:

A genre comprises a class of communicative events, the members of which share some set of communicative purposes. These purposes are recognized by the expert members of the parent discourse community, and thereby constitute the rationale for the genre. This rationale shapes the schematic structure of the discourse and influences and constrains choice of content and style. Communicative purpose is both a privileged criterion and one that operates to keep the scope of a genre as here conceived narrowly focused on comparable rhetorical action. In addition to purpose, exemplars of a genre exhibit various patterns of similarity in terms of structure, style, content and intended audience. If all high probability expectations are realized, the exemplar will be viewed as prototypical by the parent discourse community. The genre names inherited and produced by discourse communities and imported by others constitute valuable ethnographic communication, but typically need further validation (Swales, 1990: 58). 
Bhatia (2002) menciona el objetivo primordial que persigue la teoría de los géneros, lo que aclara también la necesidad de su existencia y de su uso en las lenguas de especialidad (Bhatia, 2002: 5).

to represent and account for the seemingly chaotic realities of the world; to understand and account for the private intentions of the author, in addition to socially recognized communicative purposes; to understand how language is used in and shaped by socio-critical environment; and, to offer effective solutions to pedagogical and other applied linguistic problems (Bhatia, 2002: 5).

En consonancia con este objetivo, el género era definido por Swales (1990) desde los postulados de la comunicación, como un acontecimiento comunicativo entre un grupo de personas, una comunidad, que comparte una serie de propósitos comunes sobre los que se establece la comunicación. El género es el medio por el cual se comunica esta comunidad discursiva, en el que los textos están sometidos a una forma específica y, por lo tanto, a variación dentro de cada grupo social, que dista en elementos tales como el propósito, la audiencia o la macroestructura del escrito.

Aunque la definición de Bhatia (1993: 13) de los géneros profesionales y académicos está en deuda con los trabajos de Swales (1981, 1985, 1990), difiere y amplia esta concepción basada en los factores lingüísticos y sociológicos al incorporar al nivel de construcción del género la vertiente psicológica, particularmente desde un punto de vista cognitivo:

Taking Genre, after Swales (1981b, 1985 and 1990), it is a recognizable communicative event characterized by a set of communicative purpose (s) identified and mutually understood by the members, of the professional or academic community in which it regularly occurs. Most often it is highly structured and conventionalized with constraints on allowable contributions in terms of their intent, positioning, form and functional value. These constraints, however, are often exploited by the expert members of the discourse community to achieve private intentions within the framework of socially recognized purpose (s) (Bhatia, 1993: 13). 
Para resumir, se puede afirmar que cada género es el resultado de la consecución del propósito de una comunidad concreta usando el conocimiento convencional del lenguaje y los recursos discursivos disponibles a su alcance. El propósito comunicativo se refleja en la estructuración interpretativa cognitiva del género, que representa las regularidades en la organización de este. Existe una vinculación, por lo tanto, entre el propósito comunicativo y la estructuración cognitiva típica. Estas regularidades deben ser vistas desde una perspectiva cognitiva porque reflejan las estrategias que los miembros de un discurso particular o una comunidad profesional típicamente usan en la construcción del género, así como en el logro de los propósitos específicos comunicativos.

En este sentido, Cassany (2004: 921) comenta que los géneros han de observarse desde un enfoque sociolingüístico historicista, ya que los textos están vinculados a unas prácticas de comunicación que se desarrollan en el seno de comunidades unidas socialmente por tradiciones, rutinas y reglas que se han ido configurando a lo largo de los siglos. Como consecuencia de este hecho, las comunidades son diferentes y, por lo tanto, sus manifestaciones escritas también lo son. En función de los países, una carta comercial, dice, es diferente en España, Francia o Gran Bretaña, pero del mismo modo ocurre esta variación dentro de una misma lengua entre las distintas comunidades discursivas -en Argentina y México-, aunque las cartas hispanas guarden más elementos comunes entre sí que con las de otros países. Es decir, la variación de los géneros no ocurre sólo entre distintas lenguas o propósitos, sino también a través de la propia comunidad discursiva. El mismo análisis podría aplicarse a los artículos de investigación, tesis u otros géneros académicos, profesionales o científicos.

En su artículo de 2002, Applied Genre-Analysis: a multi-perspective model, Bhatia considera el AG desde dos visiones distintas. Por una parte, entiende el género como un fiel reflejo de la complejidad de la comunicación institucionalizada, en constante mutación, interrelación e imbricación. Por otra, reconoce la utilidad del AG como una herramienta pedagógicamente efectiva y útil para el diseño de programas de lenguas. Menciona también -y este es el propósito de su artículo- que a pesar de que tradicionalmente se han presentado como posturas enfrentadas, son dos perspectivas compatibles. En ambos casos, el conocimiento del género permitirá a los estudiantes futuros profesionales- adaptarse a contextos sociales nuevos, haciendo un uso 
pragmático y retórico adecuado de la lengua. Bhatia (2002) propone un modelo de AG en el que sus elementos se solapan, en el que propone tres perspectivas que se concretan en la textual, la sociocognitiva y la sociocrítica y que a su vez se complementan entre sí (Véase Bhatia, 2002).

Este fenómeno parte de la concepción del género como una actividad multidisciplinaria en la que intervienen especialistas del campo de la lingüística, el $\mathrm{AD}$, la comunicación de expertos y retóricos, sociólogos, científicos cognitivos, traductores y publicistas, entre otros. Además de esto y ligado al concepto de la complejidad ya comentada, la sociedad -al igual que el mundo de las lenguas de especialidad- es dinámica y con propensión a una renovación constante e innovación, ya que abarca y se expresa en una gran variedad de textos en los que se multiplican los propósitos comunicativos y las formas -tanto lingüísticas como organizativas- de darles representación. Esta complejidad envuelta en la visión genérica del texto se encuentra reflejada en los siguientes postulados enunciados por Bhatia (2002: 7-8):

1. Aunque los géneros se identifican según sus rasgos convencionales, sabemos que están en continuo desarrollo (Berkenkotter and Huckin, 1995);

2. Frecuentemente encontramos patrones típicos en la textualización, aunque sabemos que los miembros expertos de las comunidades profesionales los explotan con el fin de crear nuevos patrones (Berkenkotter and Huckin, 1995);

3. Sabemos que los géneros están normalmente al servicio de los propósitos comunicativos socialmente reconocidos, aun así, frecuentemente encontramos que los géneros son utilizados para transmitir intenciones privadas (Bhatia, 1993, 1995);

4. Identificamos rasgos genéricos individuales, aunque en el mundo real se perciben frecuentemente como formas híbridas, mezclas o incrustadas en otras (Fairclough, 1993; Bhatia, 1997a, 1997b);

5. Se dan nombres típicos a los géneros; aun así, diferentes miembros de las comunidades discursivas mantienen perspectivas variables en su 
interpretación, que en ocasiones son confrontadas (Candlin \& Plum, 1999b; Bhatia, 1999b);

6. Creemos que los géneros son independientes de la variación disciplinar, aunque encontramos conflictos disciplinarios en muchos de ellos, especialmente en los géneros académicos (Bhatia, 1998b, 1999b);

7. Generalmente asociamos el análisis de género con una investigación textual típica, a pesar de que se encuentran buenos análisis a partir del uso de diferentes métodos, incluyendo el análisis textual, las técnicas etnográficas, los procedimientos cognitivos, el rigor computacional y la conciencia crítica, por mencionar solo algunos de ellos (Bhatia, 2002: 7-8).

Como puede comprobarse en estos puntos señalados por Bhatia (2002), el género es un elemento en constante progreso que está sujeto al cambio continuamente, especialmente en función a la interacción que mantiene con otros géneros en la misma o entre diferentes disciplinas. De la interacción entre las distintas características genéricas surgen nuevas formas incrustadas que sirven para dar respuesta a las nuevas realidades que se plantean en la comunicación. En su aplicación pedagógica, con el fin de conocer y superar estas trabas que imponen los textos en la comunicación social dentro de cada comunidad discursiva, Bhatia (2002) propone que se prepare al estudiante universitario para hacer frente a las demandas interactivas de la comunicación disciplinaria en la que se especializa el individuo, lo que le facilitará el ingreso en la comunidad disciplinaria a la que ha decidido acceder a través del manejo de una textualidad específica.

\section{La especialización de las lenguas con propósitos específicos}

La lengua de especialidad es una forma discursiva que plantea una terminología y unas estructuras textuales propias con una función muy específica de comunicación dentro de un grupo de individuos concretos, y que -a causa de estas peculiaridades- la distingue de otros grupos. Swales (1990: 2) atribuye al artículo de Barber de 1962 el comienzo del discurso especializado, entendido este como el uso de un lenguaje específico utilizado 
en situaciones reales y específicas en contextos académicos o profesionales. Hyland (Matsuda y otros, 2003: 165), por su parte, afirma que el concepto de género ha sido fundamental para entender el nacimiento y el desarrollo de las lenguas con propósitos específicos desde los años ochenta, así como para establecer una base empírica en la que apoyar su enseñanza tanto en L1 como en L2. De hecho, para diseñar y desarrollar tanto programas como materiales para la enseñanza de la L1 y L2, la enseñanza de lenguas con propósitos académicos y profesionales específicos (LPE), ha partido de los progresos hechos en el ámbito del AG (Bhatia, 2008: 157). En este sentido, a la altura de 1990 Swales comentaba que a causa de la pedagogía requerida para enseñar la L2, se ha profundizado más en las diferencias retóricas entre los géneros y, a partir de los errores cometidos en la didáctica de la L2, en RC: "An orientation towards providing assistance for non-native speakers has preserved a strong interest in the linguistic manifestation of rhetorical and organizational features" (Swales, 1990: 4).

Una cuestión a la que se viene prestando una atención privilegiada dentro de este campo es a la complejidad que los diversos géneros presentan en los diferentes contextos interculturales, lo que tendrá consecuencias importantes para el éxito o el fracaso pragmático de la comunicación en contextos institucionalizados y que han sido planteados desde el AD, el AG y la RC (Bhatia, 2008). Una de sus características principales, como comenta Bhatia (2008: 164), es el dinamismo al que está sometido el género. Como resultado de la evolución en las prácticas laborales y el desarrollo en el análisis de los géneros discursivos, los contextos de estudio de la LPE han ido desarrollándose igualmente de modo paralelo a estos. Si, por ejemplo, las primeras formas de $\mathrm{AG}$ estaban principalmente basadas en el estudio lingüístico del texto, éstas han ido progresando hasta marcos multidimensionales, con un énfasis especial puesto en los varios factores extratextuales en el análisis del uso del lenguaje, esto es, en cómo los miembros de la comunidad discursiva los interpretan y los utilizan tanto en contextos académicos como profesionales. Es por ello que no le resulta extraño a Bhatia (2008) que los modelos más recientes del AD y del AG hayan ido prestando hasta el presente una mayor atención a las diversas formas de contextualización. A partir de ellas Bhatia (2008: 160-165) distingue tres contextos de ampliación de la LPE, que son: 
1. los contextos multidisciplinarios en la práctica profesional, es decir, las vinculaciones entre los distintos géneros y la creación de otros nuevos a partir de estos (o subgéneros por manipulación de alguno de estos géneros).

2. la producción de discursos en LPE, es decir, la producción de textos profesionales a partir del concepto de género, tanto en formato convencional del escrito como en los nuevos medios electrónicos, destacando las formas de comunicación escritas por Internet, como el correo electrónico o el chat (Véase Cassany, 2005b). Exige la necesidad de colaboración a través de las fronteras disciplinarias.

3. la enseñanza y el aprendizaje de la traducción y la importancia de mantener o negociar la integridad del género que se está produciendo en el proceso de traducción, atendiendo a las diferencias genéricas en las distintas culturas. El reto de recrear un texto para una comunidad discursiva distinta y que el propósito sea recibido del mismo modo por la audiencia, manteniendo las intenciones que partían del texto fuente (Bhatia, 2008: 166).

Estos análisis multidimensionales o multivariables de los que venimos hablando han permitido, de igual modo, perfilar de forma más precisa cuáles son los límites disciplinarios en el terreno de los discursos académicos y cuáles son las áreas en las que se manifiesta esta variación. En este punto admite Swales (1990) la variación como una característica congénita a los géneros:

If there were only minor differences among genres there would be little need for genre analysis as a theoretical activity separable from discourse analysis, and probably no need at all for an analysis driven by applied concerns. But, of course, it turns out that genres vary significantly along quite a number of different parameters (Swales, 1990: 61-62).

De este mismo concepto se deriva la superposición existente entre disciplinas. Ante esto, Bhatia (2008) propone el abandono de una visión contemplativa para introducir el bisturí y diseccionar los géneros. Según este autor, no es posible quedarse en una mera forma estandarizada, sino que hay que atender a la complejidad en la que se entrecruzan los discursos disciplinarios desde una perspectiva multidisciplinar y multidimensional. Para ello es necesario que los miembros de las comunidades disciplinarias, en colaboración con los especialistas del lenguaje, trabajen con una perspectiva innovadora 
en las prácticas y metodologías empleadas por las distintas disciplinas en sus formatos textuales.

Como correlato a este propósito, Swales (2004: 13) hace un repaso por cómo diferentes disciplinas prefieren unos géneros con respecto a otros para expresarse: la Biología prioriza la monografía, mientras que la Antropología, la Historia, la Filosofía, la Historia del Arte y la Lingüística se transmiten más frecuentemente mediante el artículo de investigación o los libros, resultando este último el menos valorado en las áreas de ciencia e ingeniería. Sin embargo, ha de tenerse en cuenta que estos valores cambian a lo largo del tiempo, como muestra el artículo de Yadav (2010) The Decline of Conceptual Articles and Implications for Knowledge Development, con respecto al abandono de los artículos conceptuales en la disciplina del Marketing. Del mismo modo, estos textos sufrirán en su evolución modificaciones en los distintas niveles que los conforman (Swales, 2004), tanto en lo puramente lingüístico, como en lo pragmático, discursivo, sociocognitivo, etc. En este sentido, refiriéndose a la naturaleza dinámica y al rápidamente cambiante ámbito de los discursos profesionales, Swales (2004) vaticina la generación progresiva de nuevos géneros. Este autor define este fenómeno de cambio en la escritura especializada como la red de géneros (Swales, 2004: 21). Refiere que ningún género se crea de la nada, y que lo que sucede realmente es que se produce una reelaboración de los géneros precedentes mediante conexiones intertextuales, a partir de rasgos lingüísticos y retóricos. De este modo, un nuevo género discursivo no es más que la transformación de uno o varios géneros de los que toma el modelo. Algunos ejemplos que menciona Swales (2004: 21) son la inclusión y la interacción de elementos del habla en los correos electrónicos académicos, la conferencia, elementos del borrador de la investigación, diapositivas de una ponencia, apuntes, etc.

Otro aspecto destacado que afecta y amplia el contexto de la LPE es el de la revolución que supone en el campo del saber el uso de los medios tecnológicos (teléfono, fax, correo electrónico, video, televisión, Internet, etc.) en los ámbitos profesionales y académicos y la repercusión que estos tienen en el discurso escrito. Como consecuencia de la incorporación al texto de los patrones discursivos propios de estas nuevas tecnologías se han producido modificaciones en los géneros (Véase Casamasagh, 2003; 
Matsuda y otros, 2003; Cassany, 2005a, 2005b) debido al surgimiento de nuevas formas de literacidad que Cassany (2005a: 3-5) desglosa en:

- Multiliteracidad: se leen muchos textos y muy variados en breves espacios de tiempo. En Internet se salta de una página a otra, cambiando de género, idioma, tema, propósito, etc. en un breve lapso de tiempo, como en un zapping de lectura;

- Biliteracidad: este concepto insiste en la resistencia crítica y la discusión de la retórica de la segunda lengua, la búsqueda de dialogismo o mezcla cultural y la búsqueda de una textualidad coherente, de una forma mixta de retórica que deje satisfecha a la comunidad de lectores del nuevo idioma y también al autor. Esto sucede cuando se manejan dos lenguas;

- Literacidad electrónica: usamos géneros electrónicos nuevos, como el chat, el MSM, los juegos de simulación, email, webs, foros o blogs. Cada uno de estos géneros presenta nuevos parámetros pragmáticos, discursivos y verbales. La investigación sobre la literacidad electrónica estudia estos nuevos usos. En el ámbito de la didáctica de la lengua surgen propuestas de un enfoque electrónico, que sugiere que en la enseñanza de la lengua debe incluirse la enseñanza de los discursos electrónicos, en la medida que en el uso real de la lengua éstos ya ocupan un volumen importante (Cassany, 2005a: 3-5).

Concluye Cassany (2005a) con la afirmación de que la literacidad está adoptando nuevas estructuras textuales y que, como consecuencia, su estudio debe abordarse de forma urgente, con el fin de conocer sus características, con el propósito de que tanto el escritor como el lector se adapten a los nuevos requerimientos que exige la escritura en determinados géneros.

De igual modo, dentro de este panorama en continua expansión y cambio, debido a la globalización social e idiomática, ya tampoco son tan evidentes los contrastes en Retórica Contrastiva como lo eran tan solo hace unas décadas (Connor, 2001, 2002). Es un hecho probado que los textos están influidos por los de otras comunidades lingüísticas (Véase Graddol, 1997, 2006), y algunos estilos de escritura en español se aproximan a los patrones discursivos ingleses (Moreno, 1997; Albeola, 2002; Trujillo, 2002). Esto sucede especialmente con aquellos más recientes, surgidos en las últimas 
décadas por las necesidades específicas de comunicación que se le planteaban a algunos grupos sociales, y que importan modelos o técnicas foráneos cuando no existen en la propia lengua. Los jóvenes escolares y universitarios ya no acuden en busca de información a las bibliotecas para realizar sus trabajos académicos, sino que obtienen la información necesaria a su alcance con un clic de ratón, navegan por la red, crean blogs, envían correos o chatean entre sí en un interactivo intercambio de información, lo que supone un nuevo desafío para el análisis y el conocimiento de las nuevas formas discursivas que nacen de este nuevo comportamiento social. Estos nuevos usos surgidos en la era de la información, tienen que recibir una pronta respuesta con el objetivo de adaptar los formatos de los géneros a las nuevas necesidades de expresión de una sociedad cambiante y en continua transformación.

Bhatia (2008) estudia en el contexto profesional las consecuencias textuales de la mezcla, las adaptaciones y la imbricación de los géneros, sobre todo en el ámbito de la publicidad a través de cartas promocionales, campañas, folletos, etc., que cada vez resultan más complejos y variables, lo que posibilita que los profesionales creen géneros mixtos o incrustados. Así, este autor concibe que el dinamismo del género, como venimos señalando, es el factor crucial para que se continúen produciendo las transformaciones del discurso en el futuro (Bhatia, 2008: 174).

El futuro de la LPE descansa, pues, en dos aspectos importantes de la visión que he delineado aquí: (i) ser capaz de capitalizar los contextos cada vez más amplios en los que se ve que el LPE puede hacer contribuciones; y (ii) ser capaz de identificar los temas emergentes y juntar nuestros recursos para manejarlos, basándonos en sólidos principios teóricos y del modo pedagógicamente más efectivo. Si la teoría y la práctica de la LPE permanece alerta ante estos desarrollos, tiene todo el potencial para contribuir a otras áreas del uso del lenguaje en contextos académicos, profesionales e institucionales, que hasta ahora han permanecido ajenos a las preocupaciones de la disciplina (Bhatia, 2008: 174).

En relación a esto, Parodi (2007) habla de la proximidad que existe entre el continuo de textos que se entrecruzan de forma interactiva. Este hecho es, según dicho autor, el que propicia que resulte tan compleja la distinción entre los lenguajes de especialidad. De 
hecho, tras haber estudiado los textos con un análisis basado en la Lingüística del Corpus en cuatro áreas disciplinarias de conocimiento en el ámbito universitario y profesional -652 textos de Química Industrial, Ingeniería de Construcción, Trabajo Social y Psicología- afirma Parodi (2007: 153) que es casi nada lo que sabemos de los géneros escritos que circulan en la academia y en el ámbito profesional, pues están contínuamente sometidos al cambio debido a su intrínseco dinamismo. Los géneros van mutando y crecen a partir de la relación con otros, como señalaba Swales (2004) al hablar de la red de géneros. A pesar de estas disimilitudes, los discursos están muy cercanos unos de otros y se entrecruzan, como ejemplifica Parodi (2007) con el discurso técnico-científico, el profesional, el pedagógico y el institucional, pues aunque estos se han ido separando debido a la especialización que demanda su comunidad discursiva, no dejan de estar hermanados, al partir de una misma columna vertebral o "género madre" del que se originan. Sin embargo, debido a esta variación discursiva, este autor propone que no se hable de discurso disciplinar como concepto unitario, sino que resultaría más conveniente hablar de discursos disciplinarios en plural -en referencia a lo expuesto por Hyland (2000) y Bhatia (2004)- como construcción divergente del conocimiento especializado en las diferentes comunidades discursivas. En opinión de este autor, los estudios para conocer estos límites fronterizos entre las diferentes manifestaciones textuales están aún en ciernes, y sería necesario invertir una suma considerable de esfuerzos y de tiempo por parte de los investigadores del discurso para conocer mejor la relación que existe entre los textos y las características particulares que los unen y los separan.

Con respecto a las características comunes que comparten estas variaciones discursivas en relación a la lengua de especialidad de la que se generan, dentro del texto científicotécnico pueden distinguirse unos rasgos característicos que comparten las distintas variaciones discursivas que lo toman como base: un léxico común y específico, la ausencia de polisemia, sinonimia y los valores connotativos, la utilización de la función referencial del lenguaje en una búsqueda explícita de la objetividad y la despersonalización del texto, la complejidad sintáctica o el uso masivo de préstamos de otras lenguas generadoras de conocimiento lingüístico (Blanco, 2010). Una descripción similar, hecha en estos mismos términos y de forma pormenorizada en el texto de especialidad académico en español, puede consultarse en Vázquez $(2001,2004)$. 


\section{Conclusiones}

Resulta previsible que esta tendencia evolutiva y el desarrollo conceptual del texto de especialidad se vaya afianzando en las próximas décadas y que a raíz del conocimiento cada vez más especializado de las disciplinas surjan nuevos textos interrelacionados con los de otras comunidades discursivas, pero que mantendrán las peculiaridades idiosincrásicas de su disciplina, reflejadas en las distintas dimensiones que conforman el texto. Como se ha mostrado a lo largo de este artículo, el concepto de género ha venido enriqueciéndose desde los años ochenta con el aporte de disciplinas como la Sociología, la Psicología Cognitiva o la Lingüística Teórica y la Aplicada, que han ampliado el análisis lingüístico del texto para comprobar la interrelación multidimensional que existe entre los diferentes elementos que lo conforman. Como consecuencia de este hecho, se observa una progresiva adecuación y adaptación del texto a las distintas comunidades discursivas en función de los objetivos que cada una persigue y de la macroestructura, la retórica o los elementos sociopragmáticos que cada una impone.

El dinamismo es otro factor congénito al texto, que se mantendrá activo en la dirección de los cambios que se produzcan en la sociedad y, por ende, dentro de las distintas comunidades disciplinarias. Esta tendencia se potencia con la aportación de las nuevas tecnologías y de las nuevas formas de literacidad, al tiempo que se expande en la búsqueda de otras vías alternativas que expresen las necesidades de las distintas comunidades discursivas a partir de la conjunción de géneros y de las estructuras textuales cercanas entre sí, que se interrelacionan para producir nuevas formas textuales de comunicación cada vez más especializadas.

Bibliografía

Albeola, P. (2002). El folleto bancario como género: estudio contrastivo inglésespañol. Tesis doctoral. Valencia: Universidad de Valencia.

Alcaraz, E. y Martínez, M. A. (1997). Diccionario de Lingüística Moderna [2004]. Barcelona: Ariel. 
Anson, C.M. (1988). Toward a multidimensional model of writing in the academic disciplines. En: D.A. Jolliffe, ed. Advances in writing research: Writing in academic disciplines. Norwood, NJ: Ablex, 1-33.

Barber, C. L. (1962): Some measurable characteristics of modern English scientific prose. Gothenburg Studies in English 14: 21-43.

Bhatia, V. K. (1993). Analysing genre: Language use in professional settings. Londres: Longman.

Bhatia, V.K. (2002). Applied genre analysis: a multi-perspective model. Ibérica, 4, 319. http://www.aelfe.org/documents/text4-Bhatia.pdf

Bhatia, V. K. (2004). Worlds of written discourse. A genre-based view. Londres: Continuum.

Bhatia, V.K. (2008). Lenguas con Propósitos Específicos: Perspectivas cambiantes y nuevos desafíos. Revista Signos, 41 (67), 157-176.

Blanco, A. (2010). Características lingüísticas, pragmáticas y funcionales de las lenguas de especialidad, implicaciones didácticas y metodológicas. Revista Electrónica de Lingüística Aplicada, 9, 72-85.

Canagarajah, A.S. (2003). Practicing multiliteracies. Journal of Second Language Writing, 12, 156-165.

Cassany, D. (2004). La expresión escrita. En: J. Sánchez Lobato y I. Santos Gargallo, dirs. Vademecum para la formación de profesores. Enseñar español como segunda lengua (L2)/ lengua extranjera (LE). Madrid: SGEL, 917-942.

Cassany, D. (2005a). Investigaciones y propuestas sobre literacidad actual: multiliteracidad, internet y criticidad. Conferencia inaugural. Congreso Nacional Cátedra UNESCO para la lectura y la escritura, Sede Concepción. Chile: Universidad de Concepción. Disponible en: http://www2.udec.cl/catedraunesco/index.htm

Cassany, D. (2005b). Expresión escrita en L2/ ELE. Madrid: Arco/ Libros.

Connor, U. (2001). Changing Currents in Contrastive Rhetoric: New Paradigms. En: A.I. Moreno y V. Colwell, eds. Perspectivas recientes sobre el Discurso. León: Universidad de León, 27-56. 
Connor, U. (2002). New Directions in contrastive rhetoric. TESOL Quarterly, 36 (4), 493-510.

Dudley-Evans, T. (2000). Genre analysis: a key to a theory of ESP? Ibérica, 2, 3-11.

Graddol, D. (1997). The future of English? Londres: British Council.

Graddol, D. (2006). English Next. Londres: British Council.

Halliday, M.A.K. (1973). Explorations in the functions of language. Londres: Edward Arnold.

Halliday, M.A.K. (1985). An Introduction to Functional Grammar. Londres: Edward Arnold.

Hasan, R. (1989). The structure of a Text. En: M.A.K. Halliday y R. Hasan, eds. Language, Context and Text: Aspects of Language in a Social-Semiotic Perspective. Oxford: Oxford University Press.

Huckin, T. (1986). The use of discourse patterning in foreign language reading and vocabulary. Documentos de Estudios en Lingüística Teórica y Aplicada, 2 (1), $.57-75$.

Hyland, K. (2000). Disciplinary discourses: Social interactions in academic writing. Harlow, UK: Longman.

Hyland, K. (2002). Authority and invisibility: authorial identity in academic writing. Journal of Pragmatics, 34, 1091-1112.

Lackstrom, J.E. (1981). Logical argumentation: The answer to the Discussion-Problem in E.S.T. En: L. Selinker, E. Tarone, y V. Hanzeli, eds. English for academic and technical purposes: Studies in honor of Louis Trimble. Rowley, MA: Newbury House. 1981, 12-22.

Martin, J.R. (1992). English text: System and Structure. Amsterdam: John Benjamins.

Matsuda, P.K. (2003). Second language writing in the twentieth century: A situated historical perspective. En: B. Kroll, ed. Exploring the dynamics of second language writing. New York: Cambridge University Press, 15-34. 
Matsuda, P.K., Canagarajah, S., Harklau, L., Hyland, K. y Warschauer, M. (2003). Changing currents in second language writing research: a colloquium. Journal of Second Language Writing, 12, 151-179.

Moreno, A. (1997). Genre constraints across languages: causal metatext in Spanish and English RAs. English for Specific Purposes, 16 (3), 161-179.

Oliver del Olmo, S. (2004). Análisis contrastivo español/ inglés de la atenuación retórica en el discurso médico. El artículo de investigación y el caso clínico. Tesis doctoral (PhD). Barcelona: Universitat Pompeu Fabra.

Parodi, G. (2007). El discurso especializado escrito en el ámbito universitario y profesional. Revista Signos, 40 (63) 147-178.

Swales, J. (1981). Aspects of Article Introductions. Birmingham: University of Aston.

Swales, J. (1985). Episodes in ESP. Oxford: Pergamon Press.

Swales, J. (1986). Citation Analysis and Discourse Analysis. Applied Linguistics, 7 (1), 39-56.

Swales, J. (1990). Genre analysis: English in academic and research settings. Cambridge: Cambridge University Press.

Swales, J. (2004). Research genres: explorations and applications. Cambridge: Cambridge University Press.

Trimble, L. (1985). English for science and technology. A discourse approach. Cambridge: Cambridge University Press.

Trujillo, F. (2002). RC y expresión escrita. Evaluación y estudio de textos en inglés y en español, Granada: Universidad de Granada.

Van Dijk, T. A. (1977). Texto y contexto [1980] Madrid: Cátedra.

Van Dijk, T. A. (1981). Studies in the pragmatics of discourse. The Hague/ Berlin: Mouton.

Van Dijk, T A. (1993). Principles of Critical Discourse Analysis. Discourse and Society, 4 (1), 249-283.

Vázquez, G. (2001). Guía didáctica del discurso académico escrito. ¿Cómo se escribe una monografía? Madrid: Edinumen. 
Vázquez, G., (2004). La enseñanza del español con fines académicos. En: J. Sánchez Lobato y I. Santos Gargallo, dirs. Vademecum para la formación de profesores. Enseñar español como segunda lengua (L2)/ lengua extranjera (LE). Madrid: SGEL, 1129-1148.

Yadav, M. (2010). The decline of conceptual articles and implications for knowledge development. Journal of Marketing, 74, 1-19.

Recibido: 24 noviembre 2011

Aceptado: 29 enero 2014

Revisado: 11 febrero 2014

Publicado: 24 febrero 2014

Actualizado: 3 marzo 2014 